\title{
TIMELINESS OF TRAVEL-ASSOCIATED LEGIONNAIRES' DISEASE SURVEILLANCE
}

\section{LETTER TO THE EDITOR, IN RESPONSE TO THE PAPER "LEGIONELLOSIS: A WALK-THROUGH TO IDENTIFICATION OF THE SOURCE OF INFECTION", CENT EUR J PUBLIC HEALTH 2017;25(3):235-239}

\author{
Emmanuel Robesyn ${ }^{1,2}$, Lara Payne Hallström¹, Johanna Young ${ }^{1}$, Birgitta de Jong ${ }^{1}$ \\ ${ }^{1}$ European Centre for Disease Prevention and Control, Stockholm, Sweden \\ ${ }^{2}$ Karolinska Institute, Stockholm, Sweden
}

Address for correspondence: E. Robesyn, Gustav III:s boulevard 40, 16973 Solna, Sweden. E-mail: emmanuel.robesyn@ecdc.europa.eu

https://doi.org/10.21101/cejph.a5443

We would like to comment on the paper 'Legionellosis: a walk-through to identification of the source of infection' by D. Chochlakis et al. (1). The paper highlights some important aspects of the surveillance of travel-associated Legionnaires' disease. The authors do so through an interesting case report. Despite that we have a different understanding of the reporting timeline of this particular case (European Legionnaires' Disease Surveillance Network (ELDSNet) records show the case notification referred to was sent to Greece within 24 hours after the case was reported to the European Centre for Disease Prevention and Control (ECDC)), we fully support the general point from the authors on the importance of timeliness of the surveillance scheme.

The early implementation of environmental control measures in tourist accommodation can prevent further cases and, as such, be potentially life-saving for new guests. Further, the identification of likely sources, based on site assessments and water sampling results, provides the evidence needed to empower strong and targeted medium- and long-term follow-up prevention measures. These two aspects are at the very core of the travel-associated Legionnaires' disease surveillance scheme and depend largely on the timeliness of the surveillance.

ELDSNet has previously shown (2) that over half of its travelassociated clusters would remain undetected without the international collaboration of this surveillance scheme; notably when cases having stayed at the same tourist accommodation develop their symptoms upon return to different countries.

For the surveillance scheme to play its role, a chain of involved health and public health authorities, from the local to the international and back to the local level, need to work together and ELDSNet actively coordinates these efforts (3). We analysed the

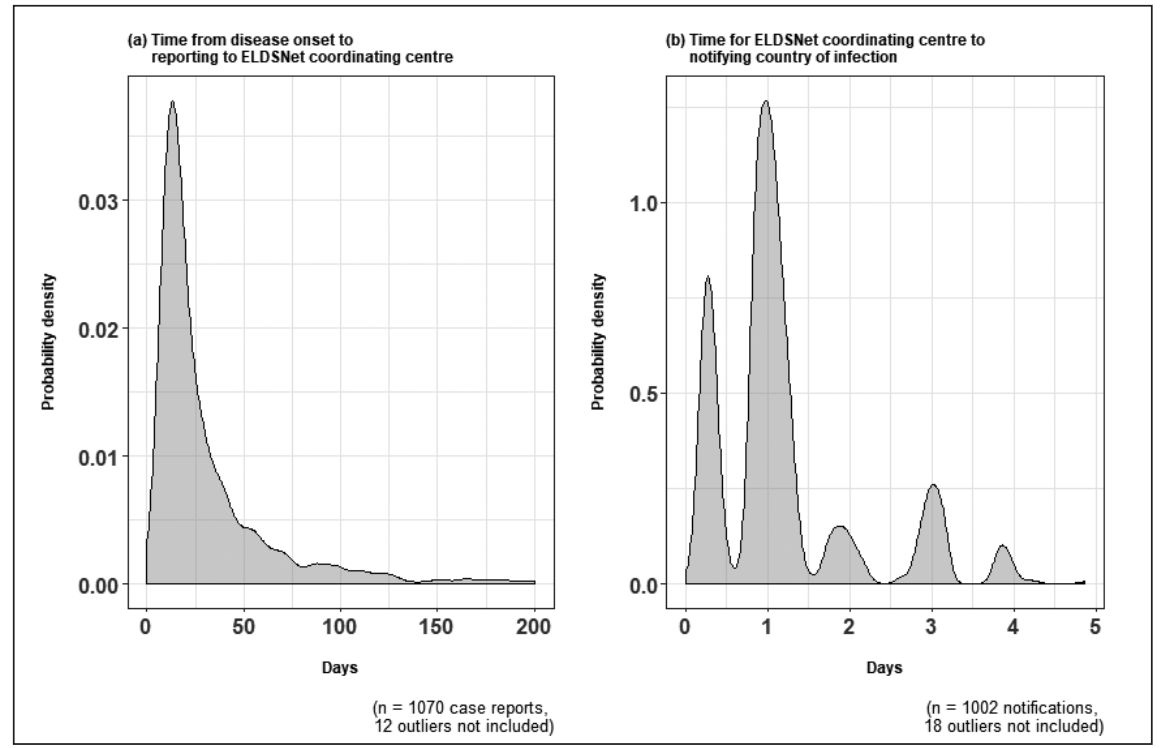

Fig.1a, 1b. Report and notification time distribution: (a) Time from disease onset to reporting to ELDSNet coordinating centre and (b) time for ELDSNet coordinating centre to notifying country of infection, EU/EEA, travel-associated Legionnaires'disease, 2016. 
time delay of two communication flows: the time from disease onset to reporting to ELDSNet, and the time for the ELDSNet coordinating centre at ECDC to send out the notification. Figure 1a, $1 \mathrm{~b}$ shows the two delay distributions. The two are not strictly comparable because they consist of different steps depending on a number of factors (including patient clinical presentation and health care seeking behaviour), and ELDSNet does not hold information about the timing of local public health authorities being notified. However, we want to put the international notification delay in perspective of the entire time it takes from cases falling ill to triggering public health action and implementation of control measures in tourist accommodation.

In 2016, the median time from date of onset to reporting to ELDSNet coordination was 19 days (Figure 1a), ranging between reporting countries from 6 to 47 days. To support cluster detection, ELDSNet expects all travel-associated Legionnaires' disease case reports to be submitted to the surveillance scheme, even when late, and this explains a long right tail in these distributions due to some late reporting. The notification delay in Figure $1 \mathrm{~b}$ shows that the large majority of notifications in 2016 were sent out the same or following day of reporting to ELDSNet. The probability that cases get reported on days two to five is small. We found that most of these cases were reported on Friday, where the time needed for surveillance database synchronisation in combination with the weekend explains the delay.
Analyses on delay in reporting are presented at the ELDSNet annual meetings and the ELDSNet Coordination Committee, and Member States have committed to further improve timeliness where possible. Such data analysis, e.g. when done by reporting country, greatly helps to pinpoint where improvements can be made.

The tight dependence of the travel-associated Legionnaires' disease surveillance relevance on timeliness underscores that this may be where the network can potentially further improve. However, we believe this real-time surveillance scheme already excels thanks to its committed reporters and direct link to public health action.

\section{REFERENCES}

1. Chochlakis D, Sandalakis V, Keramarou M, Tselentis Y, Psaroulaki A. Legionellosis: a walk-through to identification of the source of infection. Cent Eur J Public Health. 2017 Sep;25(3):235-9.

2. European Centre for Disease Prevention and Control. Surveillance report, Legionnaires' disease in Europe, 2015. Stockholm: ECDC; 2017.

3. European Centre for Disease Prevention and Control. European Legionnaires' Disease Surveillance Network (ELDSNet): Operating procedures for the surveillance of travel-associated Legionnaires'disease in the EU/ EEA. 2017. Stockholm: ECDC; 2017.

Received and accepted June 29, 2018 


\section{SUPPLEMENTS 2017}

\section{Supplement 1}

TRENDS IN HEALTH BEHAVIOUR IN CZECH SCHOOL-AGE CHILDREN: HBSC study.

The CEJPH Supplement evaluates the contribution of the Czech Republic to the cross-national Health Behaviour in School-aged Children (HBSC) study that is conducted in partnership with the World Health Organization. This cohort crosssectional questionnaire-based study is focusing on health and health-related behaviours in adolescents in the context of their social environment in their respective countries.

The main aim of the supplement is to provide evidence on various aspects of young people's health, wellbeing and related factors as found out in the Czech Republic over multiple survey years.

\section{Supplement 2}

DEVELOPMENT OF MORTALITY FROM NON-COMMUNICABLE DISEASES IN THE SLOVAK REPUBLIC AT NATIONAL AND REGIONAL LEVEL, ITS DETERMINANTS AND INTERNATIONAL COMPARISON

The CEJPH Supplement deals with the development of mortality from non-communicable diseases and its determinants in the Slovak Republic at national and regional level from 1996 to 2014, and it also offers international comparisons. 